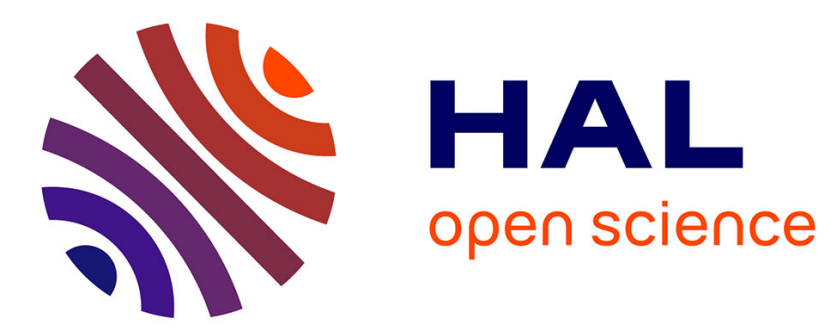

\title{
Frozen states and order-disorder transition in the dynamics of confined membranes
}

\author{
Thomas Le Goff, P. Politi, Olivier Pierre-Louis
}

\section{To cite this version:}

Thomas Le Goff, P. Politi, Olivier Pierre-Louis. Frozen states and order-disorder transition in the dynamics of confined membranes. Physical Review E , 2014, 90, pp.032114. 10.1103/PhysRevE.90.032114 . hal-02310817

\section{HAL Id: hal-02310817 https://univ-lyon1.hal.science/hal-02310817}

Submitted on 23 Sep 2021

HAL is a multi-disciplinary open access archive for the deposit and dissemination of scientific research documents, whether they are published or not. The documents may come from teaching and research institutions in France or abroad, or from public or private research centers.
L'archive ouverte pluridisciplinaire HAL, est destinée au dépôt et à la diffusion de documents scientifiques de niveau recherche, publiés ou non, émanant des établissements d'enseignement et de recherche français ou étrangers, des laboratoires publics ou privés. 


\title{
Frozen states and order-disorder transition in the dynamics of confined membranes
}

\author{
Thomas Le Goff, ${ }^{1}$ Paolo Politi, ${ }^{2,3}$ and Olivier Pierre-Louis ${ }^{1}$ \\ ${ }^{1}$ Institut Lumière Matière, UMR5306 Université Lyon 1-CNRS, Université de Lyon, 69622 Villeurbanne, France \\ ${ }^{2}$ Istituto dei Sistemi Complessi, Consiglio Nazionale delle Ricerche, Via Madonna del Piano 10, 50019 Sesto Fiorentino, Italy \\ ${ }^{3}$ INFN Sezione di Firenze, via G. Sansone 1, 50019 Sesto Fiorentino, Italy
}

(Received 17 December 2013; revised manuscript received 25 April 2014; published 15 September 2014)

\begin{abstract}
The adhesion dynamics of a membrane confined between two permeable walls is studied using a twodimensional hydrodynamic model. The membrane morphology decomposes into adhesion patches on the upper and the lower walls and obeys a nonlinear evolution equation that resembles that of phase-separation dynamics, which is known to lead to coarsening, i.e., to the endless growth of the adhesion patches. However, due to the membrane bending rigidity, the system evolves toward a frozen state without coarsening. This frozen state exhibits an order-disorder transition when increasing the permeability of the walls.
\end{abstract}

DOI: 10.1103/PhysRevE.90.032114

PACS number(s): 64.60.-i, 05.45.-a, 87.16.D-

\section{INTRODUCTION}

Two-state continuum models [1,2], such as the timedependent Ginzburg-Landau (TDGL) equation or the CahnHilliard $(\mathrm{CH})$ equation, have been widely studied as a paradigm of phase-transition dynamics in various systems, such as magnetism, liquid-liquid phase separation, or wetting. These models exhibit a phenomenology characterized by their coarsening behavior, i.e., the perpetual increase of the typical length scale of the homogeneous zones (where only one phase is present). In this paper, we propose a one-dimensional two-state continuum model inspired by adhesion of confined membranes, which gives rise to a different phenomenology without coarsening and with an order-disorder transition.

Our motivation is to investigate the adhesion dynamics of lipid membranes in biological systems. Lipid membranes are ubiquitous in living organisms. They are the main constituent of the cell membrane [3], and they also appear in stacks, e.g., in the stratum corneum of the skin [4-6]. It is therefore crucial to study their physical properties, and especially adhesion, in order to understand their biological functions. Adhesion of membranes on substrates [7-9] may include various physical ingredients, such as, e.g., van der Waals attraction and hydration forces [10], ligand-receptor pairs [11,12], interactions with the cytoskeleton [13], osmotic pressures [14], or entropic interactions [15-17]. In this paper, we do not describe these specific ingredients, rather we consider an effective adhesion potential, with a potential minimum corresponding to an equilibrium adhesion state close to the substrate [10].

The main goal of our work is to study the consequences of confinement on the nonlinear dynamics of membrane adhesion. To mimic confinement within the simplest possible setting, we consider a membrane located between two parallel flat walls. The membrane then experiences a total potential that is the sum of the adhesion potentials of the two substrates. When the distance between the walls is wider than the equilibrium distance of a supported membrane on a single wall, the membrane experiences a double-well potential with a minimum near each wall, as shown in Fig. 1. Such a double-well potential can be found in different instances in biological systems. First, in cell adhesion, this double-well potential could account for the possibility of a membrane to attach to the cytoskeleton inside the cell or to a substrate outside the cell. Moreover, in membrane stacks [14,17], each membrane may adhere to its neighbors within the stack. Furthermore, double-well potentials are also found to arise in the presence of ligands of two different lengths which enforce two different equilibrium distances in cell-cell adhesion [18]. In addition, they are also observed experimentally in the combined presence of ligands and van der Waals attraction, which induce short-range and long-range attractive potentials, respectively, and of glycocalyx and other grafted polymers, which induce a soft repulsion at intermediate scales $[19,20]$.

As a consequence of the double well, the two walls compete for the adhesion of the membrane, which is expected to adhere partially on the upper wall and partially on the lower wall. At first sight, such a decomposition into adhesion patches might exhibit some similarity with phase-separation dynamics [1], the membrane height $h(x, t)$ playing the role of the order parameter. However, in contrast to usual interfaces, which are controlled by surface tension, membranes exhibits bending rigidity $[21,22]$ : the membrane energy density is proportional to the mean curvature squared instead of being proportional to the area. We shall see in the following that this feature leads to a novel phenomenology with frozen states: adhesion patches do not grow and coarsening is absent. From an analysis of the nonlinear steady states, we argue that these frozen steady states result from the locking of bending-induced membrane oscillations into each other.

Our results could have some relevance in a recent debate about the formation and stability of finite-size adhesion domains in cell adhesion. Different studies have suggested the crucial role of the clustering of ligand-receptor pairs [23-25], of the disorder of the environment [13], of the trapping of ligands in membrane partitions [26], or of the active remodeling of the cytoskeleton [27]. We wish to stress that our model, in which adhesion is driven by a simple distancedependent free-energy potential, does not account for the full complexity of specific adhesion in cells, which involves, e.g., the attachment-detachment, diffusion, and interactions of ligand-receptor pairs, and other ingredients mentioned above. However, our results indicate a reduced set of physical ingredients that allows one to obtain finite adhesion patches: bending rigidity and confinement. 


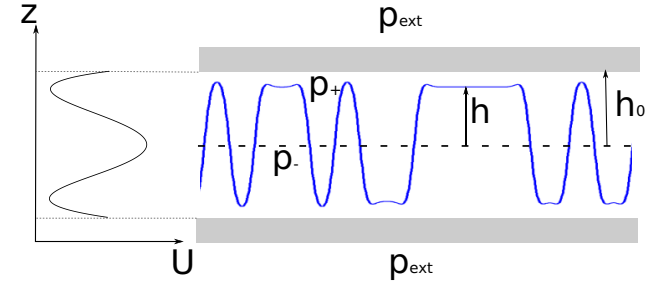

FIG. 1. (Color online) Schematics of a membrane confined between two permeable walls.

Furthermore, in order to account for the porous character of biological substrates on which the membrane may adhere, such as the cytoskeleton, collagen, or endothelial tissues, we consider walls with arbitrary permeabilities. Such a tunable permeability is also an important feature of membrane stacks in the stratum corneum [4-6]. Our modeling suggests that the spatial organization of the frozen states is controlled by the permeability of the walls. Indeed, the membrane profile exhibits a periodic ordered structure for impermeable walls, and it becomes disordered when the wall permeability is increased. This difference can be traced back to the consequences of the permeability on the initial linear instability.

In the following, we start in Sec. II with a presentation of the hydrodynamic model, and we derive a general evolution equation for a membrane between two walls in the lubrication limit. Then, in Sec. III, we consider the limits of small and large wall permeabilities. The numerical solution of these limits is discussed in Sec. IV. These results are discussed in light of a linear stability analysis in Sec. V, and of an analysis of the nonlinear steady states in Sec. VI. Finally, we summarize our results in the last section.

\section{THE HYDRODYNAMIC MODEL AND THE LUBRICATION REGIME}

We consider a membrane in a liquid confined between two parallel walls located in $z= \pm h_{0}$ (see Fig. 1). We focus on the limit of small Reynolds numbers, and the liquid obeys the Stokes equation:

$$
\nabla p_{ \pm}-\mu \Delta \mathbf{v}_{ \pm}=\mathbf{0}
$$

where the subscript \pm indicates the fluid above $(+)$ or below (-) the membrane at $z=h(x, t), p_{ \pm}(x, z)$ is the pressure, $\mu$ is the dynamic viscosity, and $\mathbf{v}_{ \pm}=\left(v_{x \pm}, v_{z \pm}\right)$ is the liquid velocity.

Next, we need to define the boundary conditions at the walls and at the membrane, which separates the upper and lower fluids. At the walls, the tangential component of the velocity vanishes because we assume no-slip conditions, while the normal component depends on wall permeability $v$ :

$$
\begin{gathered}
\left.v_{x \pm}\right|_{z= \pm h_{0}}=0, \\
\left.v_{z \pm}\right|_{z= \pm h_{0}}= \pm v\left(p_{ \pm}-p_{\text {ext }}\right),
\end{gathered}
$$

where $p_{\text {ext }}$ is a constant pressure outside the walls.

Boundary conditions at the membrane are more involved. First, following molecular-dynamics simulations on lipid membranes [28,29], we also assume no-slip at the membrane,

$$
\left.\mathbf{v}_{+}\right|_{z=h(x, t)}=\left.\mathbf{v}_{-}\right|_{z=h(x, t)} .
$$

Then, mechanical equilibrium at the membrane imposes

$$
\left(\Sigma_{+}-\Sigma_{-}\right) \cdot \mathbf{n}=\mathbf{f},
$$

where $\Sigma_{i j}=\mu\left(\partial_{i} v_{j}+\partial_{j} v_{i}\right)-p \delta_{i j}$ is the stress tensor in the fluid, $\mathbf{n}$ is the membrane normal, and $\mathbf{f}=-\delta \mathcal{E} / \delta \mathbf{r}$ is the force exerted by the membrane. This force derives from the energy

$$
\mathcal{E}=\int d s\left[\frac{\kappa}{2} C^{2}+\mathcal{U}(h)\right],
$$

where $s$ is the arc length along the membrane, $C=$ $-\partial_{x x} h /\left[1+\left(\partial_{x} h\right)^{2}\right]^{3 / 2}$ is the local membrane curvature, $\kappa$ is the bending rigidity, and $\mathcal{U}(h)$ is the double-well adhesion potential, as shown in the schematic in Fig. 1.

Finally, in order to focus on dynamics within a large contact area and to discard boundary effects, we impose periodic boundary conditions along $x$ in a large system of total length $L$.

The main approximation allowing to obtain the evolution equation for the membrane profile is the small slope approximation $\partial_{x} h(x, t) \ll 1$, while the height itself can be finite, i.e., of the order of $h_{0}$. The main lines of the derivation are reported in Appendix. Using the standard lubrication expansion [30], we obtain

$$
\begin{aligned}
\partial_{t} h= & \partial_{x}\left[-\frac{h_{0}^{3}}{24 \mu}\left(1-\frac{h^{2}}{h_{0}^{2}}\right)^{3} \partial_{x} f_{z}+\frac{3}{4} j \frac{h}{h_{0}}\left(\frac{h^{2}}{3 h_{0}^{2}}-1\right)\right] \\
& +\frac{v}{2} f_{z},
\end{aligned}
$$

where the membrane force is

$$
f_{z}=-\kappa \partial_{x}^{4} h-\mathcal{U}^{\prime}(h),
$$

and the total liquid flow rate $j$ along $x$,

$$
j=\int_{-h_{0}}^{h} d z u_{x-}+\int_{h}^{+h_{0}} d z u_{x+}
$$

obeys the differential equation

$$
-\frac{h_{0}^{3}}{3 \mu \nu} \partial_{x x} j+j=\frac{1}{2} \frac{h_{0}^{3}}{\mu} \frac{h}{h_{0}}\left(1-\frac{h^{2}}{3 h_{0}^{2}}\right) \partial_{x} f_{z} .
$$

Two remarks are in order on the above equation. First, the equation is nonlocal in space. This nonlocality is seen from the fact that $j$ obeys a time-independent differential equation (10). This constraint comes from the incompressibility of the liquid. Second, the dynamics is variational, i.e., $\partial_{t} \mathcal{E} \leqslant 0$, where the energy $\mathcal{E}$ is given by Eq. (6). In the small slope approximation, the curvature is simply $C=-\partial_{x x} h$ and

$$
\mathcal{E}=\int d x\left[\frac{\kappa}{2}\left(\partial_{x x} h\right)^{2}+\mathcal{U}(h)\right] .
$$

\section{CONSERVED AND NONCONSERVED LIMITS}

We are now going to consider two important limiting cases of Eqs. (7)-(10), which are better defined using the reduced wall permeability,

$$
\bar{v}=\frac{12 \mu \kappa^{1 / 2} v}{h_{0}^{2} \mathcal{U}_{0}^{1 / 2}},
$$


where the energy scale $\mathcal{U}_{0}$ is such that $\mathcal{U}(h)=\mathcal{U}_{0} U(H)$, where $U(H)$ is of order 1 . In the limit of large permeabilities, $\bar{v} \rightarrow \infty$, we obtain

$$
\partial_{T} H=-\partial_{X}^{4} H-U^{\prime}(H), \quad(\text { TDGL4), }
$$

where $H=h / h_{0}, X=\left[\mathcal{U}_{0} /\left(\kappa h_{0}^{2}\right)\right]^{1 / 4} x$, and $T=t v \mathcal{U}_{0} /\left(2 h_{0}^{2}\right)$. In this limit, the nonlocality induced by incompressibility vanishes and the resulting equation has a manifest nonconserved character. More precisely, Eq. (13) bears a strong resemblance to the standard time-dependent Ginzburg-Landau (TDGL) equation, $\partial_{T} H=\partial_{X}^{2} H-U^{\prime}(H)$, which describes phase separation for a nonconserved order parameter [1]. However, in Eq. (13) the linear stabilizing term is fourth-order instead of being a second-order derivative, because it physically derives from bending rigidity rather than from surface tension. For this reason, we denote Eq. (13) as "TDGL4."

In the opposite limit of impermeable walls, $\bar{v}=0$, we obtain

$$
\begin{array}{r}
\partial_{T} H=\partial_{X}\left\{\left(1-H^{2}\right)^{3} \partial_{X}\left[\partial_{X}^{4} H+U^{\prime}(H)\right]\right. \\
\left.+J H\left(\frac{H^{2}}{3}-1\right)\right\}, \quad \text { (nonlocal CH4), } \\
J=-\frac{9}{L} \int_{0}^{L} d X H\left(1-\frac{H^{2}}{3}\right) \partial_{X}\left[\partial_{X}^{4} H+U^{\prime}(H)\right],
\end{array}
$$

where the time variable now exhibits a different normalization $T=\mathcal{U}_{0}^{3 / 2} t /\left(24 \mu \kappa^{1 / 2}\right)$ and $J=18 j \mu \kappa^{1 / 4} /\left(h_{0}^{3 / 2} \mathcal{U}_{0}^{5 / 4}\right)$. For vanishing permeabilities, the resulting equation is conserved, because the (incompressible) fluid remains confined between the walls. As a consequence, the membrane evolution equation shares similarities with the Cahn-Hilliard $(\mathrm{CH})$ equation $\partial_{T} H=\partial_{X X}\left[\partial_{X}^{2} H-U^{\prime}(H)\right]$, which describes phase separation for a conserved order parameter [1,2]. However, there are several differences: (i) The fourth-order derivative in the stabilizing term. This difference was expected, in line with the nonconserved case. (ii) The membrane mobility $\sim\left(1-H^{2}\right)^{3}$ vanishes as $H \rightarrow \pm 1$ due to the well-known divergence of viscous dissipation when the membrane approaches the walls [30,31]. (iii) The nonlocal effects related to $J$. The nonlocality is now manifest in the expression of $J$ as an integral over the whole system in Eq. (15). In the following, we denote Eq. (14) as the "nonlocal CH4" equation.

\section{NUMERICAL STUDY OF MEMBRANE DYNAMICS}

As a preamble, before studying the dynamics of Eqs. (13) and (14) in extended systems, we shall recall the well-known dynamics arising from the standard TDGL and $\mathrm{CH}$ equations: the profile $H(X, T) \equiv 0$ is unstable and it develops flat regions where $H$ is approximately equal to the values of one or the other minimum of the double-well potential $U(H)$. In the language of our paper, the regions where the membrane lies in a minimum of the potential correspond to adhesion patches. The zones separating two flat regions are called kinks. Within the TDGL or CH models, pairs of kinks collide and annihilate, thereby leading to the decrease of the number of adhesion patches. The typical size $\lambda$ of these patches, therefore, exhibits endless increases in time. This process is called coarsening.
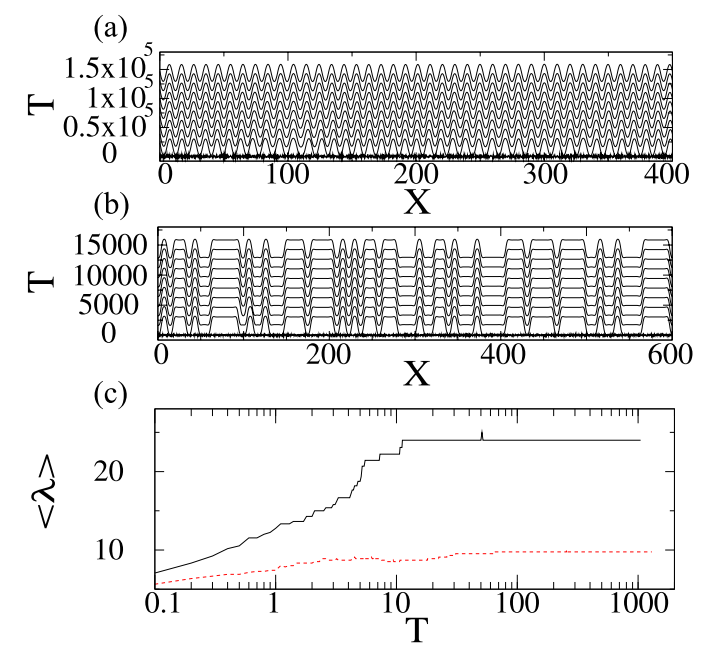

FIG. 2. (Color online) Arrested dynamics and order-disorder transition. (a) Nonpermeable case. Frozen ordered patterns obtained from the numerical solution of the $\mathrm{CH} 4$ equation, Eq. (14). (b) Permeable case. Frozen disordered patterns obtained from the numerical solution of the TDGL4 equation, Eq. (13). In (a) and (b), the vertical scale is increased by a factor $\sim 10$ for a better visibility of the membrane morphology. (c) Saturation of the spatially averaged wavelength $\langle\lambda\rangle$ as a function of time. The black solid line and the red dotted line correspond to TDGL4 Eq. (13) and CH4 Eq. (14), respectively.

In contrast, the numerical solution of TDGL4 and nonlocal $\mathrm{CH} 4$ does not exhibit any coarsening. To support this statement with numerical simulations of the evolution equations, we need to use an explicit form of the two-well potential $U$. However, in all other sections above and below, the profile of $U$ is kept arbitrary. We have chosen the standard quartic potential

$$
U_{4}(h)=-H_{m}^{2} \frac{H^{2}}{2}+\frac{H^{4}}{4},
$$

which exhibits minima at $H= \pm H_{m}$, with $H_{m}<1$. In the simulations, we use $H_{m}=0.9$.

Starting from small random initial conditions, we find that after a short transient, the membrane forms a frozen pattern, as shown in Figs. 2(a) and 2(b). To gain quantitative insights into the evolution of the system, we define the average wavelength $\langle\lambda\rangle$ as the average distance between two consecutive points obeying $h=0$ and $\partial_{x} h>0$. The plot of $\langle\lambda\rangle$ as a function of time in Fig. 2(c) shows a clear saturation after a time of the order of 10 to 30 in reduced units. Furthermore, while the frozen pattern is ordered and periodic in the presence of impermeable walls, it is clearly disordered for permeable walls. We stress that we have observed no difference between the numerical solution of nonlocal $\mathrm{CH} 4$ and Eq. (14) with $J=0$, simply denoted as $\mathrm{CH} 4$ in the following.

As a first remark on the numerical results, we indicate that simulations with other forms of the double-well potential $U$ have shown no qualitative difference in the results. However, quantitative changes can be observed. As an important example, when $H_{m} \rightarrow 1$, the conserved dynamics [Eq. (14)] slows down considerably in the late stages because the mobility term $\left(1-H^{2}\right)^{3}$ is small in the plateaus between the kinks where $H$ 
(a)

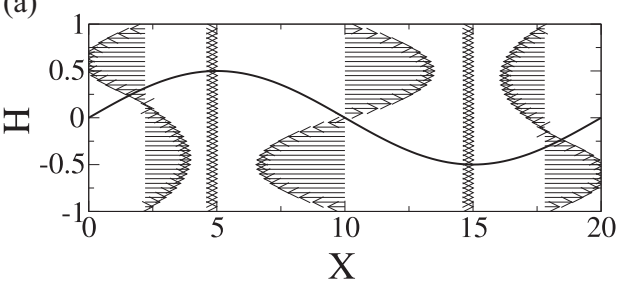

(b)

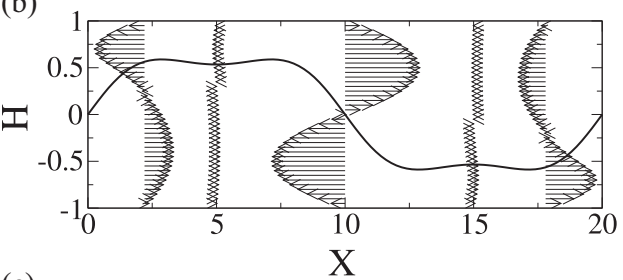

(c)

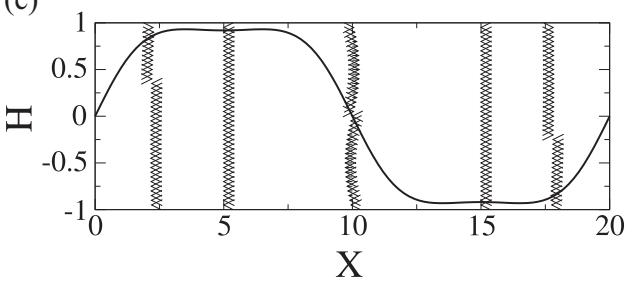

FIG. 3. Snapshots of hydrodynamics flows and membrane profile during the dynamics in the conserved case [Eq. (14)]. Horizontal arrows represent the hydrodynamic flow. (a) The initial membrane profile is a single period of a sinusoid. (b) Intermediate times. (c) The final membrane profile exhibits plateaus separated by kinks.

is close to $H_{m}$. In contrast, there is no similar effect in the nonconserved case [Eq. (13)].

Secondly, we showed that the final ordered state obtained in Fig. 2(a) for $\mathrm{CH} 4$ does not evolve further if used as an initial configuration for TDGL4. And vice versa, the final disordered state of TDGL4 in Fig. 2(b) does not evolve under $\mathrm{CH} 4$ dynamics: this is exactly what we observe from the numerical solution of the equations. This leads to two important conclusions: (i) the conserved and nonconserved equations seem to share the same stable steady states; (ii) even though distinct ordered and disordered states are robustly observed with random initial conditions, the final state may also depend on peculiar initial conditions.

Thirdly, once we have the dynamical profile of the membrane, we also have access to the full hydrodynamic flow during the evolution of the membrane using Eq. (A1). As an example, we show the flow around an initially sinusoidal membrane profile in the conserved dynamics in Fig. 3.

Finally, we observed that the normalized slopes remain finite in all simulations, i.e., $\max \left|\partial_{X} H\right| \sim 1$ at all times. As a consequence, the small slope approximation $\partial_{x} h \ll 1$ is selfconsistent: if this assumption is true initially, it remains true for all times.

In the next sections, we propose some analytical results that confirm the scenario proposed by the numerical solution of the membrane dynamics.

\section{LINEAR STABILITY ANALYSIS OF FLAT MEMBRANES}

As a summary of the results so far, Fig. 2 highlights two important features: (i) an absence of coarsening and (ii) a
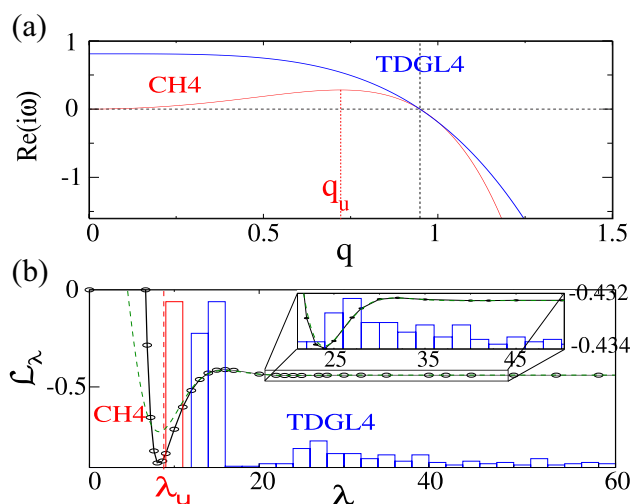

FIG. 4. (Color online) (a) Linear dispersion relation. (b) Histogram of the distances between kinks. The solid line represents the value of minus the total curvature energy in one steady-state period $\mathcal{L}_{\lambda}=-\int_{0}^{\lambda}\left[\partial_{X X} H_{\lambda}(X)\right]^{2}$, obtained numerically from the periodic double-kink solution shown in Fig. 5. As discussed in Sec. VI, $\mathcal{L}_{\lambda}$ controls the stability of the steady states. The dashed line corresponds to the approximate expression of Eq. (26) with $\mathcal{L}_{0}=-0.43225$.

frozen state that is disordered for $\bar{v}=\infty$ (TDGL4) and ordered for $\bar{v}=0$ (nonlocal $\mathrm{CH} 4$ or $\mathrm{CH} 4)$. The latter feature can be traced back to the different behaviors of the two equations with respect to small perturbations around the average height $\bar{H}$. Inserting $H(X, T)=\bar{H}+\delta H e^{i \omega T+i q X}$ with $\delta H \ll 1$ in Eq. (13), we obtain to linear order the dispersion relation for TDGL4,

$$
i \omega=-U^{\prime \prime}(\bar{H})-q^{4} \quad(\text { TDGL4) } .
$$

We remark that in the limit of permeable walls and when $U^{\prime}(\bar{H}) \neq 0$, the average height $\bar{H}$ depends on time. Hence, strictly speaking, the dynamical evolution of the Fourier modes is not exponential. However, the dispersion relation still provides a qualitative description of the unstable modes at short times for $\bar{H} \neq 0$. In addition, the linear stability analysis also provides a strictly valid description for the case $\bar{H}=0$ studied in the numerical simulations above, because $\bar{H}$ is constant in this case.

In contrast, $\bar{H}$ is always constant in the conserved equations, and the exponential time dependence of the perturbation amplitude is strictly valid in this case. The linear dispersion relation for nonlocal $\mathrm{CH} 4$ or $\mathrm{CH} 4$ (i.e., with or without the $J$ term) provides the same dispersion relation,

$$
i \omega=\left(1-\bar{H}^{2}\right)^{3} q^{2}\left[-U^{\prime \prime}(\bar{H})-q^{4}\right] \quad(\mathrm{CH} 4) .
$$

Both for permeable and impermeable walls, an instability, indicated by $i \omega>0$, appears at long wavelength when $U^{\prime \prime}(\bar{H})<0$. As seen in Fig. 4(a), while TDGL4 destabilizes all long-wavelength modes with the same growth rate $i \omega \sim$ $-U^{\prime \prime}(\bar{H})$ at $q \rightarrow 0, \mathrm{CH} 4$ exhibits a special mode at $q_{u}=$ $\left[-U^{\prime \prime}(\bar{H}) / 3\right]^{1 / 4}$ for which the growth rate is maximum. Hence, we expect initially a disordered pattern with many wavelengths in the limit of permeable walls, and an ordered pattern with a single wavelength $\lambda_{u}=2 \pi / q_{u}$ in the limit of impermeable walls. In Fig. 4(b), we have plotted the histogram of $\lambda$, the double of the distances between the zeros of $H$ in the frozen state when starting from random initial conditions. The quantity $\lambda$ is a measure of the local wavelength. For 
nonlocal (and local) $\mathrm{CH} 4$, the linear instability produces an initial periodic pattern with a single wavelength $\lambda_{u} \approx 2 \pi / q_{u}$, while for TDGL4, we indeed obtain a wide distribution of distances.

\section{STABILITY OF PERIODIC STEADY STATES}

Although linear analysis indicates when we should expect order or disorder, it does not provide insights about why the dynamics should freeze, as observed in the simulations. To gain insights on this subject, we study the stability of fully nonlinear periodic steady states. The steady states of the TDGL and $\mathrm{CH}$ equations, solutions of $\partial_{X}^{2} H-U^{\prime}(H)=0$, are known to be periodic with a single maximum in each period. For each wavelength $\lambda$, there is a unique steady state. For Eq. (7), and all its special limits TDGL4, CH4, and nonlocal CH4, the steady states obey

$$
\partial_{X}^{4} H+U^{\prime}(H)=0 .
$$

It is actually known that Eq. (19) exhibits not only periodic solutions with several maxima per period, but also an infinite number of nonperiodic solutions (chaotic along $x$ ) [32]. However, we shall show in the following that the study of periodic steady states provides a reasonable description of the nonlinear dynamics.

For this purpose, consider a family of periodic steady states $H_{\lambda}$ parametrized by the wavelength $\lambda$. We wish to study the stability of a uniform periodic steady state under long-wavelength variations of $\lambda$. Defining a macroscopic variable $\tilde{X}$ at scales much larger than $\lambda$, the total energy may be approximated as the integral on the slow variable $\tilde{X}$ of the energy density in one period,

$$
\mathcal{E}=\int \frac{d \tilde{X}}{\lambda(\tilde{X})} \int_{0}^{\lambda(\tilde{X})} d X e_{\lambda(\tilde{X})}(X),
$$

where

$$
e_{\lambda(\tilde{X})}(X)=\left[\partial_{X X} H_{\lambda(\tilde{X})}(X)\right]^{2} / 2+U\left[H_{\lambda(\tilde{X})}(X)\right]
$$

is the local energy density. We then consider a small perturbation around the average wavelength $\lambda(\tilde{X})=\bar{\lambda}+\delta \lambda(\tilde{X})$. Since $\delta \lambda(\tilde{X})$ is small, the total number $\mathcal{N}=\int d \tilde{X} / \lambda(\tilde{X})$ of steady-state periods in the system is constant, i.e., $\delta \mathcal{N}=0$, leading to the relation

$$
\lambda \int d \tilde{X} \delta \lambda(\tilde{X}) \approx \int d \tilde{X} \delta \lambda(\tilde{X})^{2}+O\left[\delta \lambda(\tilde{X})^{3}\right] .
$$

Using this relation and Eq. (19), one may then calculate the variation of total energy,

$$
\delta \mathcal{E}=\frac{\partial_{\bar{\lambda}} \mathcal{L}_{\bar{\lambda}}}{\bar{\lambda}^{2}} \int d \tilde{X}[\delta \lambda(\tilde{X})]^{2}+O\left([\delta \lambda(\tilde{X})]^{3}\right),
$$

where

$$
\mathcal{L}_{\lambda}=-\int_{0}^{\lambda}\left[\partial_{X X} H_{\lambda}(X)\right]^{2} .
$$

Since we know that the dynamics always decreases $\mathcal{E}$, i.e., $\partial_{t} \mathcal{E} \leqslant 0$, the perturbation amplitude $\int d \tilde{X}[\delta \lambda(\tilde{X})]^{2}$ must decrease if $\partial_{\lambda} \mathcal{L}_{\lambda}>0$, and it must increase if $\partial_{\lambda} \mathcal{L}_{\lambda}<0$. Hence, the periodic steady state of wavelength $\bar{\lambda}$ is stable if $\partial_{\lambda} \mathcal{L}_{\lambda}>0$ and unstable if $\partial_{\lambda} \mathcal{L}_{\lambda}<0$. This criterion shows that the stability

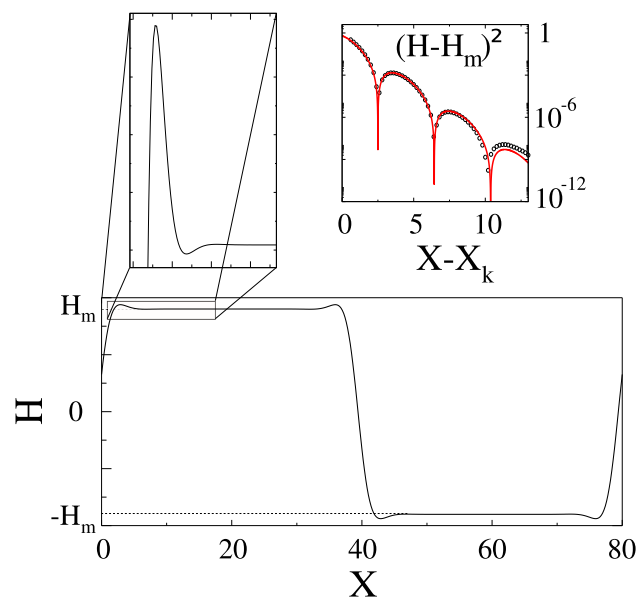

FIG. 5. (Color online) Periodic double-kink steady-state profile. The insets show a zoom on an oscillatory kink tail, and the oscillations of $\left(H-H_{m}\right)^{2}$ in log scale away from a kink. The transient dynamics leading to this periodic steady state is shown in Fig. 3.

depends only on the energy $\mathcal{E}$, and is independent of the precise kinetics. This criterion based on the energy is valid for the general Eq. (7) and its various specific limits (TDGL4, nonlocal $\mathrm{CH} 4$, or $\mathrm{CH} 4$ ).

We use a branch of steady-state solutions that provide the double-kink solution shown in Fig. 5 at long wavelengths to calculate $\mathcal{L}_{\lambda}$. Hereafter, we define a kink as a localized region of the membrane profile going from $\mp H_{m}$ for $x \rightarrow-\infty$ to $\pm H_{m}$ for $x \rightarrow+\infty$. This branch can be obtained, for example, from the relaxation with TDGL4 of an initial condition composed of a double kink with tanh profiles. In Fig. 4(b), we have plotted $\mathcal{L}_{\lambda}$ from this steady-state branch. We see that $\partial_{\lambda} \mathcal{L}_{\lambda}>0$ for the most unstable wavelength of the $\mathrm{CH} 4$ or nonlocal CH4 equations, $\lambda=\lambda_{u}$. Hence, our stability criterion explains that the periodic steady state reached by the dynamics via the linear instability of $\mathrm{CH} 4$ or nonlocal $\mathrm{CH} 4$ is frozen.

The case of the TDGL4 equation is more delicate to analyze because we start with a disordered state as discussed earlier. However, we see peaks in the histogram of Fig. 4(b) in the stable regions with $\partial_{\lambda} \mathcal{L}_{\lambda}>0$, and valleys when $\partial_{\lambda} \mathcal{L}_{\lambda}<0$. This is in agreement with a scenario in which pairs of zeros separated by a distance corresponding to $\partial_{\lambda} \mathcal{L}_{\lambda}<0$ are unstable, and the whole system finally recombines into a configuration where the distance between the zeros is in the stable regions. However, it should be noted that for large distances, no reorganization is obtained within the simulation time.

A striking feature of the stability criterion in Fig. 4(b) is its oscillatory character. These oscillations originate in the fourth-order derivative in Eq. (19), which induces an oscillatory membrane profile in the vicinity of the kinks, as shown in Fig. 5. Expanding Eq. (19) in the vicinity of the minima of potential wells at $H=H_{m}$ for $X>X_{k}$, where $X_{k}$ is the position of the kink, we find an explicit expression for the kink tails $H(X)=H_{m}+R\left(X-X_{k}\right)$, with

$$
R(\ell)=A \cos \left(\frac{\ell U_{m}^{\prime 1 / 4}}{2^{1 / 2}}+\alpha\right) \exp \left[-\frac{\ell U_{m}^{\prime 1 / 4}}{2^{1 / 2}}\right] .
$$


Here $U_{m}^{\prime \prime}=U^{\prime \prime}\left(H_{m}\right)$, and $A>0$ and $\alpha$ are constants depending on the details of the potential profile. Since we do not have an analytical expression for the full kink profile, the exact values of $A$ and $\alpha$ are unknown and depend on the precise profile of $U$. However, a simple argument provides an approximate value. Indeed, assuming that the profile $H(X)=H_{m}+R\left(X-X_{k}\right)$ with $R$ given in Eq. (25) extends beyond its domain of validity up to the center of the kink where $X \rightarrow X_{k}$, we request the continuity of $H$ at $X=X_{k}$ up to the third derivative, leading to $H\left(X_{k}\right)=0$, and $\partial_{X X} H\left(X_{k}\right)=0$. As a consequence of these assumptions, one finds $A=H_{m}$, and $\alpha=\pi$. For the specific case of the quartic potential $\mathcal{U}(H)=\mathcal{U}_{4}(H)$ with $H_{m}=0.9$, these constants can be determined numerically by fitting the profile of the tail of an isolated kink with Eq. (25), as shown in the inset of Fig. 5. We then find values that are close to the approximate predictions: $A=0.87$ and $\alpha=2.72$.

For large distances between the kinks $\lambda \gg 1$, the behavior of $\mathcal{L}_{\lambda}$ is actually dominated by the asymptotic tails of the kinks, and substituting Eq. (25) into Eq. (24), we find to leading order,

$$
\begin{aligned}
\mathcal{L}_{\lambda} \approx & \mathcal{L}_{0}+A^{2} U_{m}^{\prime \prime} \lambda \cos \left(\frac{\lambda U_{m}^{\prime \prime 1 / 4}}{2^{3 / 2}}+2 \alpha\right) \\
& \times \exp \left[-\frac{\lambda U_{m}^{\prime \prime} 1 / 4}{2^{3 / 2}}\right],
\end{aligned}
$$

where $\mathcal{L}_{0}$ is an unknown constant. This expression is in good agreement with the value of $\mathcal{L}$ obtained from the numerical profile of the steady-state branch, as shown in Fig. 4(b). As discussed above, the stability criterion is related to the sign of

$$
\begin{aligned}
\partial_{\lambda} \mathcal{L}_{\lambda} \approx & -\frac{1}{2} A^{2} U_{m}^{\prime \prime 5 / 4} \lambda \cos \left(\frac{\lambda U_{m}^{\prime \prime 1 / 4}}{2^{3 / 2}}+2 \alpha-\frac{\pi}{4}\right) \\
& \times \exp \left[-\frac{\lambda U_{m}^{\prime \prime 1 / 4}}{2^{3 / 2}}\right] .
\end{aligned}
$$

This expression shows explicitly the oscillatory character of the stability as a function of the distance between kinks.

\section{FINAL CONSIDERATIONS}

In summary, we have derived a nonlinear and nonlocal dynamical equation [see Eq. (7)] from a hydrodynamic model for a membrane separating two incompressible fluids and confined between two rigid walls; see Fig. 1. This equation has been studied numerically and analytically in the limit of large wall permeability $(\bar{v} \rightarrow \infty)$, leading to the nonconserved Eq. (13), and in the limit of vanishing wall permeability $(\bar{v} \rightarrow 0)$, leading to the conserved Eq. (14).

The bending rigidity of the membrane induces a novel class of behavior. Indeed both for small and large $\bar{v}$, the system evolves toward a frozen state, the details of which depend on the initial state. Generic, random initial configurations lead to a disordered state for large $\bar{v}$ (conserved case) and to an ordered periodic state for vanishing $\bar{v}$ (nonconserved case). The nonlocal character of the dynamics appears to be either vanishing $(\bar{v} \rightarrow \infty)$ or irrelevant $(\bar{v} \rightarrow 0)$.

The orders of magnitude of the length scales and time scales of the patterns discussed in this paper should be observable experimentally. Indeed, following Ref. [10], we consider as an example an attractive van der Waals interaction and the hydration repulsion between a membrane and a substrate. Using a gap $2 h_{0}=20 \mathrm{~nm}$ with $\bar{h}=0$, the most unstable wavelength in the case of impermeable walls (nonlocal $\mathrm{CH} 4$ ) is $\lambda_{u}=2 \pi / q_{u} \approx 350 \mathrm{~nm}$ and $t_{u} \approx 1 \times 10^{-2} \mathrm{~s}$.

Besides the need for generalization of our approach to two-dimensional membranes, one important perspective of our work is to test the robustness of the frozen states with respect to various additional physical ingredients. As an example, a membrane tension $\sigma$ can be added to the model, leading to an additional stabilizing term $\sigma \partial_{x x} h$ in the expression of the membrane force [Eq. (8)]. For large enough tensions, the oscillations in the kink tails disappear. As expected, the dynamics for large tensions is similar to that of TDGL or $\mathrm{CH}$, with logarithmic coarsening. From a simple dimensional analysis, this behavior is expected for tensions larger than $\sigma_{c} \sim\left(\mathcal{U}_{0} \kappa\right)^{1 / 2} / h_{0}$, with a prefactor of the order of 1. A detailed account of this transition confirms this prediction, and will be provided elsewhere [33]. Using once again numbers from Ref. [10], we find $\sigma_{c} \sim 10^{-2} \mathrm{~J} \mathrm{~m}^{-2}$. Values for the tension of supported membranes extracted from experiments are in the range $\sigma \approx 10^{-5}-10^{-3} \mathrm{~J}$ from Refs. [10,34]. As a consequence, the tensions observed in supported membranes are much smaller than $\sigma_{c}$, and their effects should be negligible. However, the area increase (or decrease) in the kinks during the formation (or annihilation) of adhesion patches could also lead to additional tension effects.

Other ingredients, such as potential asymmetry and noise, could also destabilize the frozen states reported here. We plan to report along these lines in the near future.

\section{ACKNOWLEDGMENTS}

We acknowledge support from Biolub Grant No. ANR-12BS04-0008 (T.L.G.,P.P. and O.P.L.), and from INFN (O.P.L.).

\section{APPENDIX: LUBRICATION LIMIT}

Here we provide the main lines of the derivation of an evolution equation for the membrane in the lubrication limit. We start with a slightly more general description as compared to the one discussed in the main text. Indeed, we describe the hydrodynamics with the full Navier-Stokes equations, including inertial effects. Consider a fluid in two dimensions, $x, z$ :

$$
\begin{aligned}
\rho\left(\partial_{t} v_{x}+v_{x} \partial_{x} v_{x}+v_{z} \partial_{z} v_{x}\right) & =-\partial_{x} p+\mu \nabla^{2} v_{x} \\
\rho\left(\partial_{t} v_{z}+v_{x} \partial_{x} v_{z}+v_{z} \partial_{z} v_{z}\right) & =-\partial_{z} p+\mu \nabla^{2} v_{z}
\end{aligned}
$$

where $\rho$ is the density of the fluid, and the other notations are defined in the main text.

We define a small parameter $\epsilon=h_{0} / \ell \ll 1$, where $\ell$ is the typical extent of the adhesion patches along $x$. We may then define dimensionless variables $X=\epsilon x / h_{0}, Z=z / h_{0}$. Following the usual procedure for the lubrication expansion [30], we also use normalized velocities $V_{X}=v_{x} / v_{0}$ and $V_{Z}=v_{z} /\left(\epsilon v_{0}\right)$, and pressure $P=\epsilon h_{0} /\left(\mu v_{0}\right)$, where $v_{0}$ is the 
typical fluid velocity. With these new variables, we obtain

$$
\begin{gathered}
\epsilon \operatorname{Re}\left(\partial_{T} V_{X}+V_{X} \partial_{X} V_{X}+V_{Z} \partial_{Z} V_{X}\right) \\
=-\partial_{X} P+\partial_{Z}^{2} V_{X}+\epsilon^{2} \partial_{X}^{2} V_{X}, \\
\epsilon^{3} \operatorname{Re}\left(\partial_{T} V_{Z}+V_{X} \partial_{X} V_{Z}+V_{Z} \partial_{Z} V_{Z}\right) \\
=-\partial_{Z} P+\epsilon^{2}\left(\partial_{Z}^{2} V_{Z}+\epsilon^{2} \partial_{X}^{2} V_{Z}\right),
\end{gathered}
$$

where $\operatorname{Re}=\rho v_{0} h_{0} / \mu$ is the Reynolds number. Assuming that $\operatorname{Re}$ is at most of order 1 , and in the lubrication approximation $\epsilon \rightarrow 0$, we obtain to leading order $-\partial_{X} P+\partial_{Z}^{2} V_{X}=0$ and $-\partial_{Z} P=0$. As a consequence, $P$ depends only on $X$, and $V_{X}$ exhibits a simple quadratic form

$$
V_{X}=\frac{Z^{2}}{2} \partial_{X} P+A Z+B,
$$

where $P, A$, and $B$ are three unknown functions of $X$ that do not depend on $Z$. Since the fluid may have different velocity profiles above and below the membrane, we obtain six unknown functions of $X$. It is convenient to define the total flow rate $J$ obeying

$$
J=\int_{-1}^{1} d Z V_{X}
$$

as a seventh unknown function of $X$.

These seven unknown functions of $X$ are obtained using the boundary conditions at the wall and at the membrane. The no-slip conditions at the walls and at the membrane, Eqs. (2) and (4), provide three equations. Then, mechanical equilibrium at the membrane, Eq. (5), leads to two additional equations. Hence, we have five equations:

$$
\begin{gathered}
\left.V_{X+}\right|_{Z=1}=0, \\
\left.V_{X-1}\right|_{Z=-1}=0, \\
\left.V_{X+}\right|_{Z=H}=\left.V_{X-}\right|_{Z=H}, \\
P_{+}-P_{-}=F_{Z}, \\
\left.\partial_{Z} V_{X+}\right|_{Z=H}=\left.\partial_{Z} V_{X-}\right|_{Z=H} .
\end{gathered}
$$

Mass conservation and the wall permeability condition, Eq. (3), provide two other equations:

$$
\begin{gathered}
\partial_{X} J=-\tilde{v}\left(P_{+}+P_{-}-2 P_{\text {ext }}\right), \\
\partial_{T} H=-\frac{1}{2} \partial_{X}\left(J_{-}-J_{+}\right)+\frac{\tilde{v}}{2}\left(P_{+}-P_{-}\right),
\end{gathered}
$$

where $\tilde{v}=v \mu \ell^{2} h_{0}^{-3}$, and the upper and lower liquid flow rates are defined as

$$
\begin{gathered}
J_{-}=\int_{-1}^{H} d Z V_{X}, \\
J_{+}=\int_{H}^{1} d Z V_{X} .
\end{gathered}
$$

Using the seven equations (A2)-(A8) provides the seven unknowns. Inserting these expressions in Eq. (A9) and going back to physical variables leads to the evolution equation of the membrane, Eq. (7).
[1] P. C. Hohenberg and B. I. Halperin, Rev. Mod. Phys. 49, 435 (1977).

[2] J. W. Cahn and J. E. Hilliard, J. Chem. Phys. 28, 258 (1958).

[3] D. Boal, Mechanics of the Cell (Cambridge University Press, Cambridge, 2002).

[4] Eur. J. Pharm. Sci. 50, 638 (2013) [(Trans)dermal drug delivery: Emerging trends to study and overcome the skin barrier].

[5] C. Das, P. D. Olmsted, and M. G. Noro, Soft Matter 5, 4549 (2009).

[6] C. Das, M. G. Noro, and P. D. Olmsted, Biophys. J. 97, 1941 (2009).

[7] C. Das, M. G. Noro, and P. D. Olmsted, Biointerphases 7, 57 (2012).

[8] M. S. Jablin, M. Zhernenkov, B. P. Toperverg, M. Dubey, H. L. Smith, A. Vidyasagar, R. Toomey, A. J. Hurd, and J. Majewski, Phys. Rev. Lett. 106, 138101 (2011).

[9] J. Daillant, E. Bellet-Amalric, A. Braslau, T. Charitat, G. Fragneto, F. Graner, S. Mora, F. Rieutord, and B. Stidder, Proc. Natl. Acad. Sci. (USA) 102, 11639 (2005).

[10] P. S. Swain and D. Andelman, Phys. Rev. E 63, 051911 (2001).

[11] D. Zuckerman and R. Bruinsma, Phys. Rev. Lett. 74, 3900 (1995).

[12] R. Lipowsky, Phys. Rev. Lett. 77, 1652 (1996).

[13] T. Speck and R. L. C. Vink, Phys. Rev. E 86, 031923 (2012).
[14] A. Hemmerle, L. Malaquin, T. Charitat, S. Lecuyer, G. Fragneto, and J. Daillant, Proc. Natl. Acad. Sci. (USA) 109, 19938 (2012).

[15] W. Helfrich, Z. Naturforsch. A 33, 305 (1978).

[16] L. B. Freund, Proc. Natl. Acad. Sci. (USA) 110, 2047 (2013).

[17] T. Auth and G. Gompper, Phys. Rev. E 88, 010701 (2013).

[18] T. R. Weikl, M. Asfaw, H. Krobath, B. Rozycki, and R. Lipowsky, Soft Matter 5, 3213 (2009).

[19] R. Bruinsma, A. Behrisch, and E. Sackmann, Phys. Rev. E 61, 4253 (2000)

[20] K. Sengupta and L. Limozin, Phys. Rev. Lett. 104, 088101 (2010).

[21] P. B. Canham, J. Theor. Biol. 26, 61 (1970).

[22] W. Helfrich, Z. Naturforsch. C 28, 693 (1973).

[23] B. G. Lorz, A.-S. Smith, C. Gege, and E. Sackmann, Langmuir 23, 12293 (2007).

[24] E. Reister-Gottfried, K. Sengupta, B. Lorz, E. Sackmann, U. Seifert, and A.-S. Smith, Phys. Rev. Lett. 101, 208103 (2008).

[25] T. Bihr, U. Seifert, and A.-S. Smith, Phys. Rev. Lett. 109, 258101 (2012).

[26] A. Kusumi, C. Nakada, K. Ritchie, K. Murase, K. Suzuki, H. Murakoshi, R. S. Kasai, J. Kondo, and T. Fujiwara, Annu. Rev. Biophys. Biomol. Struct. 34, 351 (2005). 
[27] H. Delanoë-Ayari, P. Lenz, J. Brevier, M. Weidenhaupt, M. Vallade, D. Gulino, J. F. Joanny, and D. Riveline, Phys. Rev. Lett. 93, 108102 (2004).

[28] T. J. Mueller and F. Mueller-Plathe, ChemPhysChem 10, 2305 (2009).

[29] Y. von Hansen, S. Gekle, and R. R. Netz, Phys. Rev. Lett. 111, 118103 (2013).

[30] A. Oron, S. H. Davis, and S. G. Bankoff, Rev. Mod. Phys. 69, 931 (1997).
[31] J. Happel and H. Brenner, Low Reynolds Number Hydrodynamics (Kluwer, Dordrecht, 1983).

[32] L. Peletier and W. Troy, Spatial Patterns (Birkhauser, Boston, 2001).

[33] T. Le Goff, P. Politi, and O. Pierre-Louis (unpublished).

[34] L. Malaquin, T. T. Charitat, and J. Daillant, Eur. Phys. J. E 31, 285 (2010). 